\title{
Pre-operative granulocyte/lymphocyte ratio as a predictive marker of post-operative complications in patients with colorectal cancer
}

\author{
JIRO SHIMAZAKI, TAKANOBU TABUCHI, TAKESHI NAKACHI, GYO MOTOHASHI, \\ KIYOTAKA NISHIDA, HIDEYUKI UBUKATA and TAKAFUMI TABUCHI
}

Department of Gastrointestinal Surgery, Ibaraki Medical Center, Tokyo Medical University, Ami, Ibaraki 300-0395, Japan

Received January 10, 2014; Accepted August 22, 2014

DOI: $10.3892 / 01.2014 .2669$

\begin{abstract}
The aim of the present study was to assess the clinical relevance of the pre-operative granulocyte/lymphocyte $(\mathrm{G} / \mathrm{L})$ ratio as a predictive marker of post-operative complications in patients with colorectal cancer. In total, 85 patients (59 males and 26 females; mean age, 68.9 years) underwent surgery for colorectal cancer at the Department of Surgery, Ibraki Medical Center, Tokyo Medical University (Ami, Japan), and were divided into post-operative complication and non-complication groups. Clinical data, including age, gender, body mass index, tumor localization, tumor pathological type, cancer staging, surgery time, volume of surgical bleeding, pre-operative $\mathrm{G} / \mathrm{L}$ ratio and further pre-operative laboratory data, including levels of albumin and C-reactive protein, Glasgow Prognostic Score, white blood cell count and levels of hemoglobin, creatine kinase, lactate dehydrogenase, carcinoembryonic antigen and carbohydrate antigen 19-9 were analyzed between these groups. The total post-operative complication rate was $18.8 \%$. On univariate analysis, the amount of surgical bleeding and the pre-operative $\mathrm{G} / \mathrm{L}$ ratio were significantly higher in the complication group than in the non-complication group ( $299.8 \pm 361.7$ vs. $155.6 \pm 268.6 \mathrm{ml}$, $\mathrm{P}<0.05$; and $6.73 \pm 10.38$ vs. $3.49 \pm 2.78, \mathrm{P}<0.05$, respectively). Multivariate logistic regression analysis for the risk factors of post-operative complications, determined using univariate analysis, demonstrated that the amount of surgical bleeding and the pre-operative $\mathrm{G} / \mathrm{L}$ ratio were independent risk factors of post-operative complications in patients with colorectal cancer. In conclusion, the G/L ratio may be a clinically
\end{abstract}

Correspondence to: Dr Jiro Shimazaki, Department of Gastrointestinal Surgery, Ibaraki Medical Center, Tokyo Medical University, 3-20-1 Chuo, Ami, Inasiki, Ibaraki 300-0395, Japan

E-mail: shima-j@tokyo-med.ac.jp

Key words: granulocyte/lymphocyte ratio, colorectal cancer, post-operative complication, predictive marker relevant pre-operative predictive marker for post-operative complications.

\section{Introduction}

As a result of significant improvements in surgical methods and techniques used in the treatment of colorectal cancer, the post-operative outcome of patients has improved. However, in certain cases, life-threatening complications may occur following colorectal surgery (1). The evaluation of the risk factors associated with post-operative complications in patients with colorectal cancer and the identification of prognostic indicators is required to reduce the post-operative mortality rate. Clinically, an increase in circulating leucocytes, primarily granulocytes, has been observed in patients with all advanced cancers; furthermore, the cellular immune reaction has been reported to decrease with disease progression in these patients (2). Several studies have demonstrated that the level of the granulocyte/lymphocyte $(\mathrm{G} / \mathrm{L})$ or neutrophil/lymphocyte $(\mathrm{N} / \mathrm{L})$ ratio may predict prognosis following surgery and/or chemotherapy in patients with colorectal cancer (3-8), however, no studies investigating the potential associations between the post-operative complications and pre-operative $\mathrm{G} / \mathrm{L}$ ratio have been reported. Therefore, the current study reviewed our clinical experience with surgical therapy for colorectal cancer and retrospectively analyzed the clinical relevance of the pre-operative $\mathrm{G} / \mathrm{L}$ ratio as a predictive marker of post-operative complications in patients with colorectal cancer.

\section{Materials and methods}

Between January 2011 and June 2013, 85 patients (59 males and 26 females; mean age, 68.9 years) underwent surgery for colorectal cancer at the Department of Surgery, Ibaraki Medical Center, Tokyo Medical University (Ami, Japan). No patients had received pre-operative chemotherapy. The patients were divided into post-operative complication $(n=16)$ and non-complication $(n=69)$ groups. The patient medical records were reviewed and clinical data, including age, gender, body mass index (BMI), tumor localization, tumor pathological type, cancer staging, surgery time and volume of 
Table I. Clinical data between post-operative complication and non-complication groups.

\begin{tabular}{|c|c|c|c|}
\hline Variable & Complication $(\mathrm{n}=16)$ & Non-complication $(\mathrm{n}=69)$ & P-value \\
\hline Age, years & $67.6 \pm 12.3$ & $69.2 \pm 10.3$ & $\mathrm{NS}^{\mathrm{a}}$ \\
\hline Male gender, $\mathrm{n}$ & 13 & 46 & $N S^{b}$ \\
\hline $\mathrm{BMI}, \mathrm{kg} / \mathrm{m}^{2}$ & $21.9 \pm 4.7$ & $22.5 \pm 3.9$ & $\mathrm{NS}^{\mathrm{a}}$ \\
\hline Tumor localization, $\mathrm{n}$ & & & $\mathrm{NS}^{\mathrm{b}}$ \\
\hline Colon & 7 & 41 & \\
\hline Rectum & 9 & 28 & \\
\hline Pathological tumor type, $\mathrm{n}$ & & & $\mathrm{NS}^{\mathrm{b}}$ \\
\hline Well- to moderately-differentiated & 16 & 64 & \\
\hline Poorly-differentiated & 0 & 5 & \\
\hline Cancer staging, $\mathrm{n}$ & & & $\mathrm{NS}^{\mathrm{a}}$ \\
\hline $0-\mathrm{I}$ & 2 & 20 & \\
\hline II & 4 & 23 & \\
\hline III & 3 & 10 & \\
\hline IV & 7 & 16 & \\
\hline Surgery time, min & $199.8 \pm 75.4$ & $180.2 \pm 81.4$ & $\mathrm{NS}^{\mathrm{a}}$ \\
\hline Surgical bleeding, $\mathrm{ml}$ & $299.8 \pm 361.7$ & $155.6 \pm 268.6$ & $<0.05^{\mathrm{a}}$ \\
\hline
\end{tabular}

${ }^{a}$ The Mann-Whitney U test and ${ }^{\mathrm{b}}$ Fisher's exact test were applied. BMI, body mass index; NS, not significant.

surgical bleeding were collected, in addition to pre-operative laboratory data, including levels of albumin and C-reactive protein (CRP), white blood cell (WBC) count, G/L ratio and levels of hemoglobin, creatine kinase (CK), lactate dehydrogenase (LDH), carcinoembryonic antigen (CEA) and carbohydrate antigen (CA) 19-9. The cancer staging was classified according to the degree of differentiation and the International Union Against Cancer tumor-node-metastasis classification (7th edition) (9). The Glasgow Prognostic Score (GPS) was calculated using the serum albumin and CRP levels (10). These 18 evaluative factors were compared between the groups. Written informed consent was obtained from all patients and the study was approved by the ethics committee of Ibaraki Medical Center, Tokyo Medical University (Ami, Japan).

Data are presented as the mean \pm standard deviation (SD). Stat Mate IV (ATMS Co., Ltd., Tokyo, Japan) was used to perform the statistical analysis. The Mann-Whitney U test, Fisher's exact test and the Pearson product-moment correlation coefficient were used to evaluate the correlations between variables in the univariate analysis, and a logistic regression model was used to perform multivariate analysis. $\mathrm{P}<0.05$ was considered to indicate a statistically significant difference in all tests.

\section{Results}

Of the 85 patients, $16(18.8 \%)$ developed post-operative complications; six presented with an ileus (7.1\%), five with an anastomotic leak (5.9\%), two with surgical site infection (SSI; 2.4\%), two with hepatic insufficiency (2.4\%) and one with disseminated intravascular coagulation (DIC; $1.2 \%$ ). In addition, two patients $(2.4 \%)$ who developed hepatic insufficiency and DIC, respectively, succumbed to the disease within 60 days of the surgery.

Table I summarizes the clinical data for the complication and non-complication groups. The volume of surgical bleeding was significantly higher in the complication group $(299.8 \pm 361.7 \mathrm{ml})$ compared with the non-complication group $(155.6 \pm 268.6 \mathrm{ml} ; \mathrm{P}<0.05)$. Although the surgery time was longer in the complication group compared with the non-complication group (199.8 \pm 75.4 vs. $180.2 \pm 81.4 \mathrm{~min}$, respectively), the difference was not statistically significant. Furthermore, the differences in complications associated with age, gender, BMI, tumor localization, tumor pathological type and cancer staging were not statistically significant (Table I).

Table II reveals the comparison of the pre-operative laboratory data, including levels of albumin and CRP, WBC count, GPS, G/L ratio and levels of hemoglobin, CK, LDH, CEA, and CA19-9, between the complication and non-complication groups. The G/L ratio was significantly higher in the complication group compared with the non-complication group $(6.73 \pm 10.38$ vs. $3.49 \pm 2.78$, respectively; $\mathrm{P}<0.05)$. However, no statistically significant difference was identified in the complications associated with differences in levels of pre-operative albumin and CRP, WBC count, GPS and levels of hemoglobin, CK, LDH, CEA and CA19-9 (Table II).

The multivariate logistic regression analysis, conducted using the complication risk factors determined by univariate analysis (the amount of surgical bleeding and the G/L ratio), revealed that the volume of surgical bleeding and the $\mathrm{G} / \mathrm{L}$ ratio 
Table II. Pre-operative laboratory data (mean \pm standard deviation) between post-operative complication and non-complication groups.

\begin{tabular}{lccc}
\hline Variable & Complication $(\mathrm{n}=16)$ & Non-complication $(\mathrm{n}=69)^{\text {P-value }^{\mathrm{a}}}$ \\
\hline Albumin, g/dl & $3.63 \pm 0.63$ & $3.91 \pm 0.60$ & $\mathrm{NS}$ \\
CRP, mg/dl & $2.42 \pm 3.28$ & $1.15 \pm 3.02$ & $\mathrm{NS}$ \\
GPS & $0.68 \pm 0.87$ & $0.46 \pm 0.81$ & $\mathrm{NS}$ \\
WBC count, per $\mu \mathrm{l}$ & $7143 \pm 1969$ & $6462 \pm 1912$ & $\mathrm{NS}$ \\
G/L ratio & $6.73 \pm 10.38$ & $3.49 \pm 2.78$ & $<0.05$ \\
Hemoglobin, g/dl & $12.5 \pm 10.4$ & $11.9 \pm 2.4$ & $\mathrm{NS}$ \\
CK, IU/l & $94.0 \pm 89.2$ & $90.9 \pm 71.2$ & $\mathrm{NS}$ \\
LDH, IU/l & $270.5 \pm 178.7$ & $227.8 \pm 164.9$ & $\mathrm{NS}$ \\
CEA, ng/ml & $393.7 \pm 1089.8$ & $111.3 \pm 464$ & $\mathrm{NS}$ \\
CA19-9, U/ml & $165.6 \pm 527.6$ & $627.2 \pm 2799.3$ & $\mathrm{NS}$
\end{tabular}

${ }^{a}$ The Mann-Whitney U test was applied. CRP, C-reactive protein; GPS, Glasgow Prognostic Score; WBC, white blood cell; G/L, granulocyte/ lymphocyte; CK, creatine kinase; LDH, lactate dehydrogenase; CEA, carcinoembryonic antigen; CA, carbohydrate antigen; NS, not significant.

Table III. Multivariate analysis of the independent risk factors for post-operative complications.

\begin{tabular}{lccc}
\hline Variables & Odds ratio & 95\% Confidence interval & P-value $^{\mathrm{a}}$ \\
\hline Surgical bleeding, $\mathrm{ml}(<100,100-200,200<)$ & 1.912 & $1.018-3.587$ & 0.043 \\
G/L ratio $(<2.5,2.5-3.5,3.5<)$ & 2.180 & $1.112-4.274$ & 0.023
\end{tabular}

${ }^{a}$ Multivariate logistic regression was applied. G/L, granulocyte/lymphocyte.

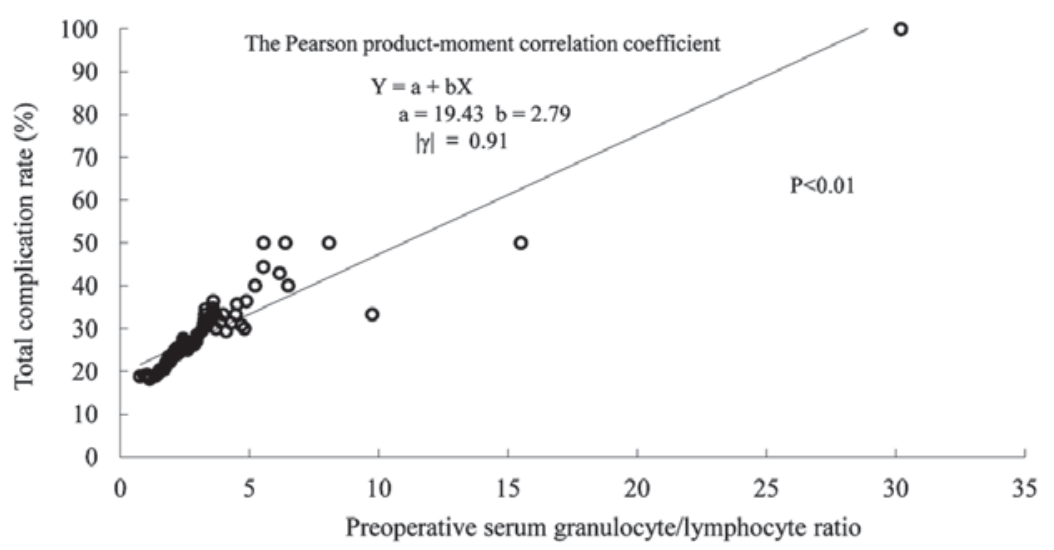

Figure 1. Linear regression established from the comparison between the pre-operative granulocyte/lymphocyte ratio levels and the total post-operative complication rate.

were independent risk factors of post-operative complications in patients with colorectal cancer (surgical bleeding: Odds ratio, 1.912; 95\% confidence interval, 1.018-3.587; $\mathrm{P}=0.043$; and $\mathrm{G} / \mathrm{L}$ ratio: Odds ratio, 2.180; 95\% confidence interval, 1.112-4.274; $\mathrm{P}=0.023$; Table III). Fig. 1 shows the statistical regression line between the pre-operative $\mathrm{G} / \mathrm{L}$ ratio levels and the total post-operative complication rate, with a statistically significant correlation $(\mathrm{P}<0.01$; Fig. 1). The post-operative complication rate could be predicted from the pre-operative $\mathrm{G} / \mathrm{L}$ ratio level.

\section{Discussion}

Post-operative complications in colorectal cancer may result in functional impairments or even fatal outcomes for the patient, and also lead to an increase in medical costs associated with an extension of the hospital stay (11-13). Predicting the development of post-operative complications is useful in the perioperative management of patients, beginning pre-operatively and continuing into the post-operative period, and an 
early response to post-operative complications has contributed to a decrease in post-operative mortality rates $(14,15)$.

The three most frequent post-operative complications of surgery for colorectal cancer are ileus, anastomotic leak and SSI. Chapuis et al (16) reported that the incidence of a post-operative ileus was $14.0 \%$ and that the risk factors included male gender, comorbidity with respiratory diseases, emergency surgery and if the surgery had been performed in $>3 \mathrm{~h}$. In addition, the incidence of anastomotic leaks and SSI have been reported to be $1.5-11.5 \%$ and $11.7-15.7 \%$, respectively, with risk factors including high BMI, history of laparotomy, advanced-stage cancer and malnutrition (17-21). Yasunaga et al (22) reported that the incidence of complications in Japanese subjects following gastrointestinal surgery was at its lowest when the BMI was $\sim 23.0 \mathrm{~kg} / \mathrm{m}^{2}$, but that it was high when the BMI was $\leq 18.5$ or $\geq 30.0 \mathrm{~kg} / \mathrm{m}^{2}$. In the current study, the mean BMI of the subjects in the complication and non-complication groups was $\sim 22.0 \mathrm{~kg} / \mathrm{m}^{2}$, therefore indicating that there was no association between BMI and the development of post-operative complications. The nutritional assessment of patients with cancer is significant in improving the prognosis. Ishizuka et al $(23,24)$ reported the GPS to be a post-operative prognostic factor in patients with colorectal cancer. However, in the current study, no association between the GPS and post-operative complications was identified. This is possibly due to the fact that the study was comprised of patients with a wide range of colorectal cancers, from early-stage to advanced-stage cancers with distant metastasis. A comparison between the complication group and non-complication group showed no statistically significant difference in GPS, as although the levels of CRP tended to be high in the complication group, there was almost no difference in the albumin levels. An albumin level of $\leq 3.5 \mathrm{~g} / \mathrm{dl}$ is defined as low (and thus associated with increased risk) by GPS (10). In patients with colorectal cancer, problems associated with oral intake due to intestinal transit disorders, advanced-stage cancer or cachexia associated with distant metastases may result in hypoalbuminemia (25). Therefore, this indicates that if the control group was comprised of only patients with highly-advanced colorectal cancers, the GPS may be a risk factor for post-operative complications (26).

The present study examined 18 factors and focused on the pre-operative $\mathrm{G} / \mathrm{L}$ ratio in the blood of patients with colorectal cancer, and further determined the correlation between the G/L ratio and post-operative complications. A correlation between the prognosis and the $\mathrm{G} / \mathrm{L}$ or $\mathrm{N} / \mathrm{L}$ ratios has been reported in various types of cancers (27-29). Clinically, patients with cancer occasionally present with an elevated WBC count, particularly an elevated granulocyte count, and a decreased lymphocyte count. When the progression and proliferation of the cancer is chronic and biologically invasive, it is accompanied by functional impairments due to localized tissue hemorrhage, ischemia or necrosis and causes various types of immune inflammatory responses. These responses include an elevated WBC count, particularly an elevated granulocyte count, the immunosuppression of lymphocytes, elevated levels of inflammatory cytokines, such as interleukin (IL)-6, and acute phase reactants, such as $\mathrm{CRP}$, a negative nitrogen balance due to increased decomposition of muscle proteins, and the inhibition of albumin synthesis in the liver. The reactions are caused by a cytokine network composed of IL-6, IL-1 and tumor necrosis factor- $\alpha(30,31)$. IL-6 is a lectin, which is produced by T-cells, neutrophils, macrophages and tumor cells, and a cytokine that controls humoral immunity $(32,33)$. The extent of biological invasion can be determined by the IL- 6 levels; in colorectal cancers, IL-6 is reportedly associated with the progression of cancer and prognosis $(34,35)$. We previously reported that a high $\mathrm{G} / \mathrm{L}$ ratio prior to surgery is a risk factor associated with the prognosis of patients with colorectal cancer, and that a correlation exists between the $\mathrm{G} / \mathrm{L}$ ratio prior to surgery and the IL- 6 concentration $(8,36)$. The findings of the present study suggest that a high $\mathrm{G} / \mathrm{L}$ ratio prior to surgery is a risk factor for post-operative complications in patients with colorectal cancer. Taking the previous findings into consideration, a high $\mathrm{G} / \mathrm{L}$ ratio prior to surgery in patients with colorectal cancer indicates high pre-operative IL-6 levels, i.e., a biological invasion, which is believed to cause post-operative complications. In addition, the present study also confirmed that the amount of surgical bleeding is a risk factor for post-operative complications. The volume of surgical bleeding reflects the damage to the biological tissue, which is accompanied by IL-6 production, and is considered to be strongly associated with post-operative complications $(37,38)$.

Among the 18 factors that were examined in the present study, only the pre-operative $\mathrm{G} / \mathrm{L}$ ratio and the volume of surgical bleeding were confirmed as risk factors for post-operative complications. Of these two factors, the $\mathrm{G} / \mathrm{L}$ ratio allows for the pre-operative prediction of post-operative complications; furthermore, the G/L ratio is simple, easy to measure and is likely to aid in the future surgical management of patients with colorectal cancer. The findings of this study raise issues that remain to be discussed, particularly those pertaining to the types of post-operative complications for which the $\mathrm{G} / \mathrm{L}$ ratio has specificity as a risk factor. To address these issues, further studies comprising a larger number of patients are required in the future.

In conclusion, to reduce the post-operative complications in patients with colorectal cancer, it is essential to reduce the possible amount of surgical bleeding. The pre-operative G/L ratio may be a clinically relevant pre-operative predictive marker in these patients.

\section{Acknowledgements}

The authors would like to thank Enago (www.enago.jp) for reviewing the English language of the original manuscript.

\section{References}

1. Whistance RN, Forsythe RO, McNair AG, et al; Core Outcomes and iNformation SEts iN SUrgical Studies- ColoRectal Cancer Working Group: A systematic review of outcome reporting in colorectal cancer surgery. Colorectal Dis 15: e548-e560, 2013.

2. Coussens LM and Werb Z: Inflammation and cancer. Nature 420: 860-867, 2002.

3. Walsh SR, Cook EJ, Goulder F, Justin TA and Keeling NJ: Neutrophil-lymphocyte ratio as a prognostic factor in colorectal cancer. J Surg Oncol 91: 181-184, 2005.

4. Halazun KJ, Aldoori A, Malik HZ, et al: Elevated preoperative neutrophil to lymphocyte ratio predicts survival following hepatic resection for colorectal liver metastases. Eur J Surg Oncol 34: 55-60, 2008.

5. Chua W, Charles KA, Baracos VE and Clarke SJ: Neutrophil/lymphocyte ratio predicts chemotherapy outcomes in patients with advanced colorectal cancer. Br J Cancer 104: 1288-1295, 2011. 
6. Ding PR, An X, Zhang RX, et al: Elevated preoperative neutrophil to lymphocyte ratio predicts risk of recurrence following curative resection for stage IIA colon cancer. Int J Colorectal Dis 25 $1427-1433,2010$.

7. Hung HY, Chen JS, Yeh CY, et al: Effect of preoperative neutrophil-lymphocyte ratio on the surgical outcomes of stage II colon cancer patients who do not receive adjuvant chemotherapy. Int J Colorectal Dis 26: 1059-1065, 2011.

8. Shimazaki J, Goto Y, Nishida K, et al: In patients with colorectal cancer, preoperative serum interleukin-6 level and granulocyte/lymphocyte ratio are clinically relevant biomarkers of long-term cancer progression. Oncology 84: 356-361, 2013.

9. Sobin LH, Gospodarowicz MK and Wittekind C (eds): TNM Classification of Malignant Tumors. 7th edition. Wiley-Blackwell Oxford, 2009

10. Forrest LM, McMillan DC, McArdle CS, Angerson WJ and Dunlop DL: Evaluation of cumulative prognostic scores based on the systemic inflammatory response in patients with inoperable non-small-cell lung cancer. Br J Cancer 89: 1028-1030, 2003.

11. Jean-Claude M, Emmanuelle P, Juliette $\mathrm{H}$, et al: Clinical and economic impact of malnutrition per se on the postoperative course of colorectal cancer patients. Clin Nutr 31: 896-902, 2012 .

12. Lee L, Li C, Landry T, Latimer E, Carli F, Fried GM and Feldman LS: A systematic review of economic evaluations of enhanced recovery pathways for colorectal surgery. Ann Surg 259: 670-676, 2014.

13. Damle RN, Cherng NB, Flahive JM, Davids JS, Maykel JA, Sturrock PR, Sweeney WB and Alavi K: Clostridium difficile Infection After Colorectal Surgery: A Rare but Costly Complication. J Gastrointest Surg: Aug, 2014 (Epub ahead of print)

14. Kwag SJ, Kim JG, Kang WK, Lee JK and Oh ST: The nutritional risk is a independent factor for postoperative morbidity in surgery for colorectal cancer. Ann Surg Treat Res 86: 206-211, 2014.

15. Pommergaard HC, Gessler B, Burcharth J, Angenete E, Haglind E and Rosenberg J: Preoperative risk factors for anastomotic leakage after resection for colorectal cancer: a systematic review and metaanalysis. Colorectal Dis 16: 662-671, 2014

16. Chapuis PH, Bokey L, Keshava A, et al: Risk factors for prolonged ileus after resection of colorectal cancer: an observational study of 2,400 consecutive patients. Ann Surg 257: 909-915, 2013.

17. Telem DA, Chin EH, Nguyen SQ and Divino CM: Risk factors for anastomotic leak following colorectal surgery: a case-control study. Arch Surg 145: 371-376, 2010

18. Aytac E, Lavery IC, Kalady MF and Kiran RP: Impact of obesity on operation performed, complications, and long-term outcomes in terms of restoration of intestinal continuity for patients with mid and low rectal cancer. Dis Colon Rectum 56: 689-697, 2013.

19. Itatsu K, Sugawara G, Kaneoka Y, et al: Risk factors for incisional surgical site infections in elective surgery for colorectal cancer: focus on intraoperative meticulous wound management. Surg Today 44: 1242-1252, 2014.

20. Takakura Y, Hinoi T, Egi H, et al: Procalcitonin as a predictive marker for surgical site infection in elective colorectal cancer surgery. Langenbecks Arch Surg 398: 833-839, 2013.

21. Bot J, Piessen G, Robb WB, Roger V and Mariette C: Advanced tumor stage is an independent risk factor of postoperative infectious complications after colorectal surgery: arguments from a case-matched series. Dis Colon Rectum 56: 568-576, 2013.

22. Yasunaga $\mathrm{H}$, Horiguchi $\mathrm{H}$, Matsuda $\mathrm{S}$, et al: Body mass index and outcomes following gastrointestinal cancer surgery in Japan. Br J Surg 100: 1335-1343, 2013.
23. Ishizuka M, Nagata $H$, Takagi K, Horie $T$ and Kubota $K$ : Inflammation-based prognostic score is a novel predictor of postoperative outcome in patients with colorectal cancer. Ann Surg 246: 1047-1051, 2007.

24. Ishizuka M, Nagata H, Takagi K, Iwasaki Y and Kubota K: Inflammation-based prognostic system predicts postoperative survival of colorectal cancer patients with a normal preoperative serum level of carcinoembryonic antigen. Ann Surg Oncol 19: 3422-3431, 2012

25. Lai CC, You JF, Yeh CY, Chen JS, Tang R, Wang JY and Chin CC: Low preoperative serum albumin in colon cancer: a risk factor for poor outcome. Int J Colorectal Dis 26: 473-481, 2011.

26. Ishizuka M, Nagata H, Takagi K, Iwasaki Y and Kubota K: Inflammation-based prognostic system predicts survival after surgery for stage IV colorectal cancer. Am J Surg 205: 22-28, 2013 .

27. Mohri Y, Tanaka K, Ohi M, Yokoe T, Miki C and Kusunoki M: Prognostic significance of host- and tumor-related factors in patients with gastric cancer. World J Surg 34: 285-290, 2010.

28. Sarraf KM, Belcher E, Raevsky E, Nicholson AG, Goldstraw P and Lim E: Neutrophil/lymphocyte ratio and its association with survival after complete resection in non-small cell lung cancer. J Thorac Cardiovasc Surg 137: 425-428, 2009.

29. Cho H, Hur HW, Kim SW, Kim SH, Kim JH, Kim YT and Lee K: Pre-treatment neutrophil to lymphocyte ratio is elevated in epithelial ovarian cancer and predicts survival after treatment. Cancer Immunol Immunother 58: 15-23, 2009.

30. Dinarello CA: Proinflammatory and anti-inflammatory cytokines as mediators in the pathogenesis of septic shock. Chest 112: 321S-329S, 1997.

31. Malicki S, Winiarski M, Matlok M, et al: IL-6 and IL-8 responses of colorectal cancer in vivo and in vitro cancer cells subjected to simvastatin. J Physiol Pharmacol 60: 141-146, 2009.

32. Ardestani SK, Inserra P, Solkoff D and Watson RR: The role of cytokines on tumor progression: A review. Cancer Detect Prev 23: 215-225, 1999.

33. Esfandi F, Mohammadzadeh Ghobadloo S and Basati G: Interleukin-6 level in patients with colorectal cancer. Cancer Lett 244: 76-78, 2006.

34. Galizia G, Orditura M, Romano C, et al: Prognostic significance of circulating IL-10 and IL-6 serum levels in colon cancer patients undergoing surgery. Clin Immunol 102: 169-178, 2002

35. Belluco C, Nitti D, Frantz M, et al: Interleukin-6 blood level is associated with circulating carcinoembryonic antigen and prognosis in patients with colorectal cancer. Ann Surg Oncol 7: $133-138,2000$

36. Tabuchi T, Shimazaki J, Satani T, et al: The perioperative granulocyte/lymphocyte ratio is a clinically relevant marker of surgical stress in patients with colorectal cancer. Cytokine 53: 243-248, 2011

37. Kvarnström A, Swartling T, Kurlberg G, Bengtson JP and Bengtsson A: Pro-inflammatory cytokine release in rectal surgery: comparison between laparoscopic and open surgical techniques. Arch Immunol Ther Exp. (Warsz) 61: 407-411, 2013.

38. Tang Y, Xia XF, Zhang Y, et al: Establishment of an experimental mouse model of trauma-hemorrhagic shock. Exp Anim 61: 417-425, 2012. 